\title{
Global description of patterns far from onset: a case study
}

\author{
N. Ercolani ${ }^{a}$, R. Indik ${ }^{a}, *$ A.C. Newell ${ }^{a}$, T. Passot $^{b}$ \\ ${ }^{a}$ Department of Mathematics, University of Arizona, Tucson, AZ 85721, USA \\ ${ }^{\mathrm{b}}$ CNRS, Observatoire de la Côte d'Azur, B.P. 4229, 06304 Nice Cedex 4, France
}

\begin{abstract}
The Cross-Newell phase diffusion equation $\tau(k) \Theta_{\mathrm{T}}=-\nabla \cdot \vec{k} B(k), \vec{k}=\nabla \Theta,|\vec{k}|=k$, and its regularization describe patterns and defects far from onset in large aspect ratio systems with translational and rotational symmetry. In this paper we show how director field solutions of this equation can be used to describe features of global patterns. The ideas are illustrated in the context of a non-trivial case study of high Prandtl number convection in a large aspect ratio, shallow, elliptical container with heated sidewalls, for which we also have the results of simulation and experiment.
\end{abstract}

(c) 2003 Elsevier B.V. All rights reserved.

Keywords: Patterns; Defects; Convection

\section{Introduction}

A macroscopic description of convection patterns far from the onset of the convective instability still presents a considerable challenge for both physicists and mathematicians. The path we have followed to tackle this problem is to study the phase diffusion equation that results from the elimination of the complex three-dimensional structure of the microscopic roll pattern and that focuses on the large-scale dynamics of the phase gradient, which is perpendicular to the rolls.

We recently established [1] rigorous proofs for the existence and form of solutions to the regularized phase diffusion equation in the limit where the inverse aspect ratio $\varepsilon$ (ratio of the roll wavelength to the typical size of the container) goes to zero. The basis of this work was first to remark that solving the phase diffusion equation amounts to solving a mini-

\footnotetext{
* Corresponding author.

E-mail address: indik@acms.arizona.edu (R. Indik).
}

mization problem for an energy $E$ which is the sum of two terms, one associated with roll bending and the other with the mismatch between the local and the preferred wave number. Generally pattern forming systems are not gradient flows. However in the high Prandtl number limit the Oberbeck-Boussinesq equations behave as if they were [2]. A second point was to study a separate, although closely related, problem defined by equating the two components of the energy. This self-dual reduction leads to solutions which are also solutions of the original phase diffusion equation when the Gaussian curvature of the graph of the phase vanishes. For many of the observed structures in patterns, the curvature does vanish except in the neighborhood of certain points or curves. In general, however, the solutions of the self-dual equations are not solutions of this phase equation but they do provide, for each $\varepsilon$, an upper bound on the energy. The remarkable point is that, for a model form of the phase equation valid when the wave number is everywhere close to the selected one, the self-dual equation can be linearized 
and leads to explicit solutions whose limits as $\varepsilon$ goes to zero, are well controlled. In special cases, a lower bound for the energy can be found, that equals the upper bound and thus provides an explicit minimizer (not necessarily unique).

One of the limitations of this work has to do with the fact that locally changing the phase gradient $\nabla \theta$ into $-\nabla \theta$ does not change the physical rolls. However, the manner in which the energy is defined associates a non-zero cost with such a reversal of the local wave number. Therefore, the solutions that have been found are possibly not the true minimizers if one defines the minimization problem in a space where the sign phase ambiguity is taken into account. Such a solution could for example be realized in terms of a two-sheeted Riemann surface, the branch cut being located on the line where this sign reversal occurs. The goal of this paper is to study the patterns obtained when the container has an elliptical lateral boundary which is heated in order to impose a constant phase on this boundary. Our theory of the phase diffusion equation leads, in the $\varepsilon \rightarrow 0$ limit, to rolls which are solutions of the eikonal equation $|\nabla \theta|=1$. In this roll pattern, the rolls come in parallel to the boundary and meet at a grain boundary (see Fig. 1; see also [3]). The main question is whether or not this solution is realized in experimental or numerical patterns. Several comparisons are made, first with one of the simplest toy models for infinite Prandtl number convection, the Swift-Hohenberg equation

$W_{t}+\left(\nabla^{2}+1\right)^{2} W-R W+W^{3}=0$,

where $W$ represents, for example, the temperature, and $R$ represents the stress parameter deviation from onset. We also compare our predictions with numerical simulations of the Oberbeck-Boussinesq equations [4] and finally with experiments [5].

The most interesting feature is to study how the sign ambiguity in the wave vector of the pattern leads, in the numerical solutions of the model equation, to a solution with an energy possibly smaller than that of the eikonal solution. It turns out that the solution "prefers" to transform a grain boundary with sharp angles into pieces of straight rolls connected with dislocations.

In Section 2, the phase diffusion equation and its regularization are introduced, together with the energy and the linear stability properties. Section 3 briefly describes the main results of our previous work [1]. In Section 4, we introduce the self-dual equation and the exact phase grain boundary (PGB) solution. Section 5 is a summary of the procedure to obtain weak solutions of the self-dual equation (steepest descent method) and the description of the eikonal solutions. In Section 6 we introduce the notion of twist associated with director fields (unsigned vectors) and explain in detail the limitations of our previous results. An argument is presented in Section 7 to predict

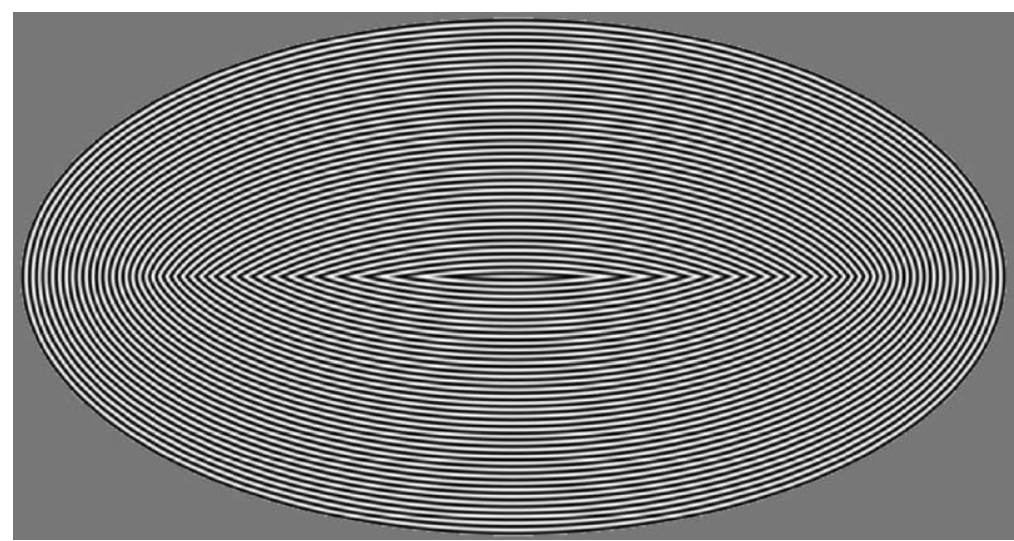

Fig. 1. The eikonal solution has rolls that are parallel to the elliptical boundary. This pattern propagates inward with fixed roll spacing. This leads to a phase grain boundary (PGB) in the middle. 
the critical angle at which a PGB becomes unstable towards the formation of dislocations. Sections 8 and 9 , respectively, describe the numerical simulations of the Swift-Hohenberg equation and the comparisons with simulations of the Oberbeck-Boussinesq equation and laboratory experiments. Some concluding remarks are made in Section 10.

\section{Background}

Let us consider a straight roll solution of the Oberbeck-Boussinesq [6], or the Swift-Hohenberg (SH) equations, of the form

$w(x, y, t)=F(\theta)=\Sigma A_{n}(k) \cos n \theta$.

$F(\theta)$ is a $2 \pi$ periodic function of a phase $\theta$ whose gradient $\vec{k}$ is the constant wave vector of the pattern. An important point, related to the question of twist discussed in Section 6, is to remark that the microscopic field is unchanged on the reversal of $\theta, \theta \rightarrow-\theta$. Next, we seek nearby solutions in the form

$w(x, y, t)=F\left(\frac{1}{\varepsilon} \Theta\right)+\varepsilon w_{1}+\varepsilon^{2} w_{2}+\cdots$

for which the local phase $\Theta$ varies over large spatial and temporal scales defined as $\vec{X}=\varepsilon \vec{x}, T=\varepsilon^{2} t$. In particular, the wave vector $\vec{k}=\nabla \theta=\nabla_{\vec{X}} \Theta=(f, g)$, changes by order 1 over distances of $(1 / \varepsilon) \lambda$, where $\lambda$ is the wavelength and $\varepsilon, 0<\varepsilon \ll 1$ is the inverse aspect ratio of the system. For the modulated solution, the amplitudes $A_{n}$ are still slaved to the local wave number $k=|\nabla \theta|$ via $A_{n}=A_{n}(k)$.

Because the original system is translationally invariant, the variation of $w$ about $F(\theta)$ has a non-trivial null space and the non-homogeneous equation for the corrections $w_{1}, w_{2}, \ldots$ to $F(\theta)$ must satisfy solvability conditions. These express the fact that the right-hand sides of these equations must lie in the range of the linear operator obtained by linearizing $w$ about the exact solution $F(\theta)$. To dominant order in $\varepsilon$ [7],

$\tau(k) \Theta_{\mathrm{T}}=-\nabla \cdot \vec{k} B(k)$.

In Eq. (2.3), $\tau(k)>0, k B(k)$ is a cubic like curve shown in Fig. 2 which is zero at the left and right

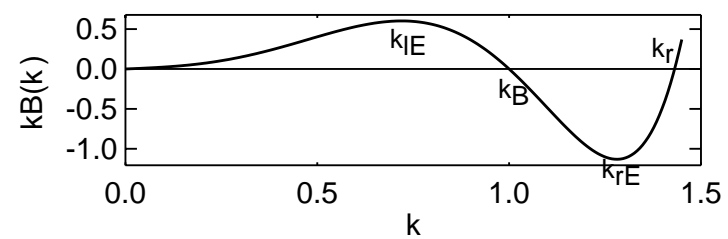

Fig. 2. Plot of a typical graph of $k B(k)$, There is a zero at $k_{\mathrm{B}}, k_{\mathrm{rE}}$ labels the right Eckhaus instability boundary, while $k_{\mathrm{IE}}$ labels the left Eckhaus instability boundary.

ends of the region of existence of (2.1) and is zero at a point $k_{\mathrm{B}}$ between. The overall shape, one maximum, one interior zero, and one minimum of $k B$ is universal for a broad class of microscopic systems. The spatial part of Eq. (2.3) becomes

$$
\begin{aligned}
\nabla \cdot \vec{k} B(k)= & \left(B+\frac{f^{2}}{k} B^{\prime}\right) \Theta_{X X}+\frac{2 f g}{k} B^{\prime} \Theta_{X Y} \\
& +\left(B+\frac{g^{2}}{k} B^{\prime}\right) \Theta_{Y Y} .
\end{aligned}
$$

It is a quasilinear second-order operator in $\Theta$ and is only negative definite when both $B$ and $(k B)^{\prime}$ are negative.

This occurs in the interval $\left(k_{\mathrm{B}}, k_{\mathrm{rE}}\right)$ known as the Busse balloon. Outside this region, Eq. (2.3) is ill-posed. When the wave number $k$ of the straight roll pattern lies to the left of $k_{\mathrm{B}}$, the rolls lose their resistance to bending (this is the zigzag instability). A regularization term is thus required in Eq. (2.3) to describe the resulting dynamics. Moreover, the stationary phase diffusion equation admits multivalued solutions [1] which can only relate to the physically relevant solutions if one adds a regularization coming from the contributions of the next orders of the asymptotic expansion. The dominant correction comes at order $\varepsilon^{3}$ and can be approximated by a linear term of the form $\varepsilon^{2} \eta \nabla^{4} \Theta$. After changing the definition of $\varepsilon$ to absorb the constant $\eta$, the regularized equation reads

$\tau(k) \Theta_{\mathrm{T}}=-\nabla \cdot \vec{k} B(k)-\varepsilon^{2} \nabla^{4} \Theta$.

The bi-Laplacian of the phase, $\varepsilon^{2} \nabla^{4} \Theta$, provides the necessary bending resistance to arrest and saturate the roll bending due to the zigzag instability and leads to a stationary line defect which is called a phase grain boundary (PGB). Across the PGB, the pattern wave 
vector $\vec{k}$ changes abruptly (over distances of the roll wavelength).

Considering a compact domain $\Omega$ with smooth boundary, one easily finds that Eq. (2.5) is essentially a gradient flow

$\tau(k) \Theta_{\mathrm{T}}=-\frac{\delta F}{\delta \Theta}$

with

$F[\Theta]=\int_{\Omega}\left(\frac{1}{2} G^{2}(k)+\frac{1}{2} \varepsilon^{2}\left(\nabla^{2} \Theta\right)^{2}\right) \mathrm{d} \vec{X}$,

and

$G^{2}(k)=-2 \int_{k_{\mathrm{B}}}^{k} k B(k) \mathrm{d} k$.

In $F[\Theta]$, the $G^{2}$ term is zero when $k=k_{\mathrm{B}}$, the preferred wave number. It represents the wave number mismatch energy density while the $\varepsilon^{2}\left(\nabla^{2} \Theta\right)^{2}$ term represents the bending energy density. The system would like to relax to the state where $k=k_{\mathrm{B}}$ everywhere. In interesting circumstances, such as the problem posed in Section 1, this cannot be the case and patches of planform where $k=k_{\mathrm{B}}$ are mediated by line and point defects.

Before analyzing the minimization problem for the energy (2.7), we emphasize the sense in which equation (2.5) is universal. First, we have assumed that there are no other slow modes than the one associated with the translational invariance of the system. In particular, we exclude cases (such as in small to moderate Prandtl number convection) where a mean flow, driven by roll curvature gradients, is present. Second, we have assumed that no short scale instability (e.g. a cross-roll instability) can develop and trigger a modification to the local roll planform.

\section{Description of asymptotic minimizers for $\mathrm{RCN}$}

For $k$ close to $k_{\mathrm{B}}, G^{2}$ is approximated to second order by $\left(k^{2}-k_{\mathrm{B}}^{2}\right)^{2}$. Choosing $k_{\mathrm{B}}=1$, the RCN (regularized Cross-Newell) free energy divided by $\varepsilon$ becomes

$$
E^{\varepsilon}[\Theta]=\int_{\Omega}\left(\varepsilon(\nabla \cdot \vec{k})^{2}+\frac{1}{\varepsilon}\left(k^{2}-1\right)^{2}\right) \mathrm{d} \vec{X} .
$$

Our goal is to study the asymptotic form, as $\varepsilon \rightarrow 0$, of the minimizers $\Theta^{\varepsilon}$ of $E^{\varepsilon}[\Theta]$ within the family of locally gradient vector fields $\vec{k}=\nabla \Theta$ for which the phase $\Theta$ belongs to the class

$\mathcal{A}^{\varepsilon}=\left\{\Theta \in H^{2}(\Omega) ; \Theta\left|\Omega=\alpha^{\varepsilon}(s), \frac{\partial \Theta}{\partial n}\right|_{\Omega}=\beta^{\varepsilon}(s)\right\}$,

where $\alpha^{\varepsilon}(s)$ and $\beta^{\varepsilon}(s)$ are two sequences of smooth bounded functions of the boundary arclength $s$ which are, respectively, uniformly convergent in $\varepsilon$.

The energy associated with the variational problem (3.1) has the form of the well-known GinzburgLandau functional [1]. The difference is that in the $\mathrm{RCN}$ variational problem this energy is restricted to vector fields which are gradient. We are able to say something about the asymptotic limit of these minimizers, in the class of $\mathcal{A}^{\varepsilon}$ coming from Dirichlet viscosity boundary conditions (these will be described in the next section). We summarize here what is known about this limit.

\section{Theorem 3.1.}

(a) As $\varepsilon \rightarrow 0$, the minimizers $\Theta^{\varepsilon} \rightarrow \Theta^{0}$ in $H^{1}(\Omega)$, where $\Theta^{0}$ solves the eikonal equation $\left|\nabla \Theta^{0}\right|=$ 1. Defects are, therefore, supported on locally one-dimensional sets which are a union of locally rectifiable curves. Let $\Sigma$ denote this total defect locus.

(b) The asymptotic minimal energy is bounded:

$\liminf _{\varepsilon \rightarrow 0} E\left(\Theta^{\varepsilon}\right) \leq \frac{1}{3} \int_{\Sigma}\left|\left[\nabla \Theta^{0}\right]\right|^{3} \mathrm{~d} s$,

where $\left[\nabla \Theta^{0}\right]$ is the jump in $\vec{k}^{0}$ across $\Sigma$ and $\mathrm{d} s$ is the element of arclength.

(c) In the class of cases when $\Sigma$ is a single straight line segment, the previous inequality becomes an equality; i.e., the upper bound is tight.

Details about the proofs of these results may be found in [1]. In that work we show that $\Theta^{\varepsilon}$ converges weakly to $\Theta^{0}$; however, more recent results on these types of minimization problems [8-10] enable one to improve this to the strong convergence stated above. 
We will construct some explicit examples of eikonal solutions in Section 5.

These one-dimensional defect loci are what we have called phase grain boundaries. Our bound on the asymptotic minimum energy indicates that the bending term $\int_{\Omega}|\nabla \vec{k}|^{2}$ in this free energy will diverge as $1 / \varepsilon$. This is a major increase in energetic cost compared to the $\log (1 / \varepsilon)$ cost of the original Ginzburg-Landau problem whose defects are point vortices. We reiterate that this difference is due to the fact that, in comparison to Ginzburg-Landau, the minimization for $\mathrm{RCN}$ is done over the smaller class of irrotational vector fields; i.e., those $\vec{k}$ which are gradient.

\section{Self-dual test functions}

Although it is straightforward to conclude the existence of the minimizers $\Theta^{\varepsilon}$ of $E^{\varepsilon}[\Theta]$, it is far from straightforward to calculate them (they are solutions of a fourth-order variational problem). Except in extremely special instances this has not, as far as we know, been done.

In earlier papers [1,11,12], we had proved that self-dual (or anti-self-dual) solutions, namely phase functions $\Theta(X, Y)$ which satisfy

$\varepsilon \nabla^{2} \Theta= \pm \sqrt{G^{2}}$

are solutions of the Euler-Lagrange equation for $F[\Theta]$,

$\varepsilon^{2} \nabla^{4} \Theta+\nabla \cdot \vec{k} B(k)=0$,

provided the Hessian (proportional to the Gaussian curvature of the graph of $\Theta$ ) vanishes; i.e.,

$\Theta_{X X} \Theta_{Y Y}-\Theta_{X Y}^{2}=0$.

The motivation for considering solutions of the self-dual equation come from the observation that, for many of the structures observed in convection patterns (PGBs, almost everywhere in the neighborhoods of dislocations and disclinations), the free energy is equipartitioned between the wave number mismatch and the bending energy components. In the weak bending limit we proved [11] that this equipartition was a fact in general; i.e., solutions of the associated self-dual equations were also solutions of the fourth-order variational equations. Although this is not the case here, because of the Hessian obstruction, it turns out that the self-dual solutions are either a good approximation (in the small $\varepsilon$ limit, the Hessian has its support at point defects [11] and along curved line defects) or, at worst, serve as good test functions for bounding the free energy in the $\varepsilon \rightarrow 0$ limit. This is the fundamental idea underlying our analysis. Because they solve a second-order PDE, one can get precise estimates on the asymptotic behavior of self-dual solutions in the limit as $\varepsilon \rightarrow 0$. For $G^{2}=\left(k^{2}-1\right)^{2}$, which is a good quantitative approximation near $k=1$, and a good qualitative match for $k_{\mathrm{IE}}<k<k_{\mathrm{rE}}$, Eq. (4.1) becomes

$\varepsilon \nabla^{2} \Theta= \pm\left(1-(\nabla \Theta)^{2}\right)$.

The transformation

$\Theta=\varepsilon \ln \Psi$

linearizes (4.4) as

$\varepsilon^{2} \nabla^{2} \Psi-\Psi=0$.

The straight roll solution of (4.6) is

$\Psi=\mathrm{e}^{\vec{k} \cdot \vec{X} / \varepsilon}, \quad k=1 \quad$ or $\quad \Theta=\vec{k} \cdot \vec{X}$.

The regularized PGB solution is simply the sum of two exponentials

$\Psi=\mathrm{e}^{\vec{k}_{+} \cdot \vec{X} / \varepsilon}+\mathrm{e}^{\vec{k}_{-} \cdot \vec{X} / \varepsilon}$,

where $\vec{k}_{ \pm}=(\cos \varphi, \pm \sin \varphi)$. The corresponding phase has gradient

$\nabla \Theta=\vec{k}=\left(\cos \varphi, \sin \varphi \tanh \left(\frac{Y}{\varepsilon} \sin \varphi\right)\right)$.

The free energy of the roll solution is zero. For the exactly self-dual regularized PGB solution (its Gaussian curvature is zero), the self-duality allows us to express the free energy as twice one of its terms:

$$
\begin{aligned}
E^{\varepsilon} & =\frac{2}{\varepsilon} \int_{\Omega}\left(1-k^{2}\right)^{2} \mathrm{~d} \vec{X} \\
& =\frac{2}{\varepsilon} \int_{\Omega} \sin ^{4} \varphi \operatorname{sech}^{4}\left(\frac{Y}{\varepsilon} \sin \varphi\right) \mathrm{d} X \mathrm{~d} Y
\end{aligned}
$$


which, if $\varphi$ is constant in $X$, gives

$\lim _{\varepsilon \rightarrow 0} E^{\varepsilon}=\frac{8 L}{3} \sin ^{3} \varphi$,

This coincides with the general result stated in Theorem 3.1.

We now use these self-dual solutions to explore how one might uncover configurations which would minimize (3.1) on $\Omega$ within the class $\mathcal{A}^{\varepsilon}$ of functions $\Theta$ satisfying boundary conditions $\left.\Theta\right|_{\partial \Omega}=\alpha(s)$, independent of $\varepsilon$, and $\beta^{\varepsilon}(s)=\left.\partial_{\nu} \Theta_{\mathrm{SD}}^{\varepsilon}\right|_{\partial \Omega}$, where $\Theta_{\mathrm{SD}}^{\varepsilon}$ is the solution of (4.4) corresponding to the boundary values $\alpha(s)$.

In [9] we show that as long as $\alpha(s)$ has a Lipschitz constant less than $1, \beta^{\varepsilon}(s)$ is uniformly convergent and therefore satisfies our admissibility conditions for the class $\mathcal{A}^{\varepsilon}$. In the next section we will observe that within such an admissible class of boundary conditions, the self-dual solutions limit to viscosity solutions of the unregularized phase equation which, in our present model, is just the eikonal equation. For this reason we refer to these boundary conditions as Dirichlet viscosity boundary conditions.

\section{Weak solutions}

For admissible Dirichlet viscosity boundary conditions, described at the end of the previous section, we consider the sequence

$\Theta_{\mathrm{SD}}^{\varepsilon}=\varepsilon \ln \Psi^{\varepsilon}$,

where $\Psi$ satisfies the Helmholtz equation (4.6) with boundary value $\mathrm{e}^{\alpha(s) / \varepsilon}$. It can be shown that $\lim _{\varepsilon \rightarrow 0} \Theta_{\mathrm{SD}}^{\varepsilon}$ is the unique continuous viscosity solution $v$ to

$|\nabla v|^{2}-1=0 \quad$ on $\quad \Omega,\left.\quad v\right|_{\partial \Omega}=\alpha(s)$.

By a steepest descent calculation [1] we give a prescription for constructing the gradient of this viscosity solution which is the appropriate object to consider when making comparisons between the self-dual solutions and minimizing sequences of the RCN energy

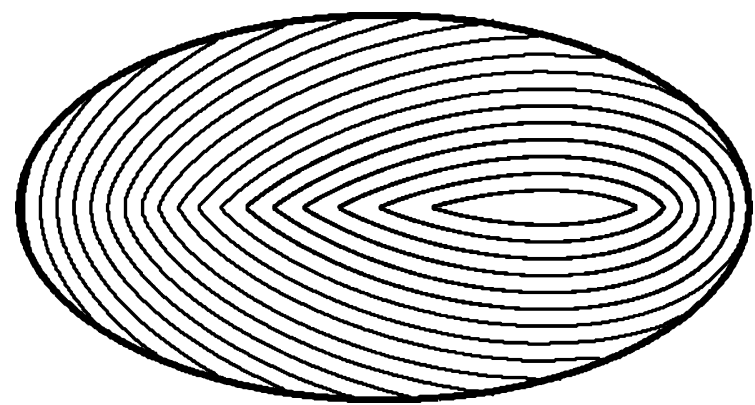

Fig. 3. Eikonal solution with non-constant boundary conditions, and horizontal defect locus.

$E^{\varepsilon}$. This prescription is

$\nabla v(\vec{X})=-\frac{\vec{X}-\vec{X}(\bar{s})}{|\vec{X}-\vec{X}(\bar{s})|}$

where $\vec{X}(s)$ is the arclength parameterization of $\partial \Omega$ and $\bar{s}$ is the point on the boundary where the expression $|\vec{X}-\vec{X}(s)|-\Theta(\vec{X}(s))$ attains a minimum. Except on a set of measure 0 in $\Omega$, this minimum point is unique and simple. The variational equations for $\bar{s}$ are

$\left(\frac{\vec{X}-\vec{X}(\bar{s})}{|\vec{X}-\vec{X}(\bar{s})|}-\nabla v(\vec{X}(\bar{s}))\right) \cdot \vec{X}^{\prime}(\bar{s})=0$.

Fig. 3 illustrates the application of this construction for the boundary data given by restricting on $\partial \Omega$ the linear function $m x, x=X / \varepsilon$ for $m=0.5$. The wave vector $\vec{k}=\nabla v=(\vec{X}(\bar{s})-\vec{X}) /|\vec{X}(\vec{s})-\vec{X}|$ has magnitude 1 everywhere. The defect locus is a straight line segment along the $x$-axis and hence is within the class to which part 3 of Theorem 3.1 applies.

Another example, and one which is pertinent for the remainder of this paper is to consider again an elliptical domain but with constant boundary data. In fact we can take $\alpha \equiv 0$. This corresponds to the boundary of the domain being a roll. In a physical setting, let us suppose that the boundary is uniformly heated so that $\theta=0$ and the temperature $F(\theta)$ is maximal there. Then the construction is the obvious one of locating the next temperature maximum a distance of $2 \pi$ inward, along the boundary normal. This construction will fail as the normals meet on a line segment along the major axis (see Fig. 1). Regularization results in a 
PGB along that segment; it is centered about the origin between two points which are the curvature centers of the ellipse and which we refer to as terminal disclinations. Associated with each point on this open segment there are two distinct points on the boundary which have the same distance to the given point on the segment. Therefore for $\vec{X}$ on (or within a wavelength distance of) the line segment joining the two terminal disclinations, from the steepest descent argument, $\Psi(\vec{X})$ is well approximated as the sum of two exponentials

$$
\begin{aligned}
\Psi(\vec{X}) \approx & \exp \left(\frac{1}{\varepsilon} \vec{k}_{1} \cdot\left(\vec{X}-\vec{X}^{(1)}(\bar{s})\right)\right) \\
& +\exp \left(\frac{1}{\varepsilon} \vec{k}_{2} \cdot\left(\vec{X}-\vec{X}^{(2)}(\bar{s})\right)\right),
\end{aligned}
$$

where $k_{j}=\left(\vec{X}^{(j)}(\bar{s})-\vec{X}\right) /\left|\vec{X}-\vec{X}^{(j)}(\bar{s})\right|$, and $\vec{X}^{(j)}(\bar{s}), j=1,2$, are the two minimizing boundary points for $\vec{X}$ on the line segment joining the two terminal disclinations. It is natural to regard this as a modulated PGB solution. This example will be discussed in further detail in Section 8.

\section{Multiple values and twist}

Because the variational problem (3.1) studied in [1] considers only variations over fields which are conservative, it cannot realize certain types of defects, such as disclinations, which are commonly seen in pattern-forming systems far from threshold. The reason is topological. Modulation equations such as $\mathrm{CN}$ or RCN are derived under the assumption that the field is locally gradient. Away from defects, striped patterns locally look like the level curves of a function such as $\cos (\theta)$. The phase $\theta$ itself need not be well defined (for instance, $\theta \rightarrow-\theta$ leaves the local pattern unaltered). The existence of these ambiguities is intimately related to the types of defects that can occur. For instance, wave vector fields $(f, g)$ with $f-\mathrm{i} g=z^{1 / 2}$ or $z^{-1 / 2}$, where $z=x+\mathrm{i} y$ give examples of harmonic vector fields for which there is not a single-valued potential $\theta$. Traversing a closed circuit counterclockwise about the origin of these fields one would find that the wave vector returns to itself but rotated by $\mp \pi$, in contrast
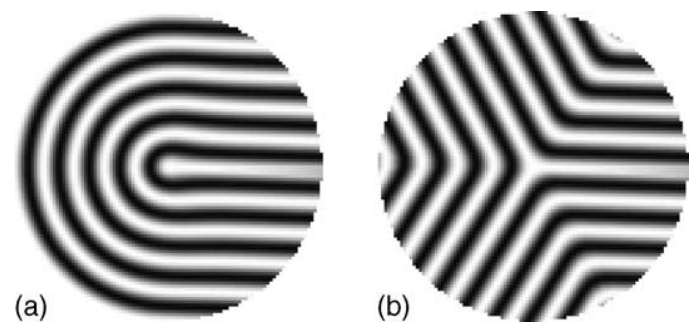

Fig. 4. (a) Convex disclination. (b) Concave disclination numerically calculated as solutions of the RCN equation.

to the conservative fields $z$ and $z^{-1}$ where traversing the closed circuit would yield a rotation of $\pm 2 \pi$. The defects of the former fields which are locally but not globally gradient are referred to as disclinations. The best way to think about this is to regard these fields as director fields, that is to say unoriented vector fields.

To include such fields, one needs to consider spaces consisting of double-valued vector functions on $\Omega$, the double-valuedness corresponding to the orientational ambiguity of director fields. One way to do this concretely would be to consider single-valued functions on domains which are Riemann surfaces rather than simply connected planar domains. In [11], we carried out such numerical and analytical studies on a double cover of a disc $\Omega$ with vectorial boundary data whose winding number is half integral. In this way, we were able to obtain stationary solutions of the RCN equation corresponding to concave (V) and convex (X) disclinations, which are the canonical point defects of two-dimensional patterns (see Fig. 4). These are ubiquitous in natural patterns, necessarily involve director fields $\pm \vec{k}$ and, as we shall shortly see, play a central role in finding the true minimizers of (3.1) in the convection context.

One important consequence of the double-valuedness of the phase is that the class of functions that was considered in the preceding sections is too small. Minimizing vector field configurations may, and often will be unstable to director field perturbations.

An examination of some computed solutions to the Swift-Hohenberg equation, or the Boussinesq equations or of experiments of Rayleigh-Bénard convection shows that these systems seem to produce solutions which have disclinations. In addition, while 


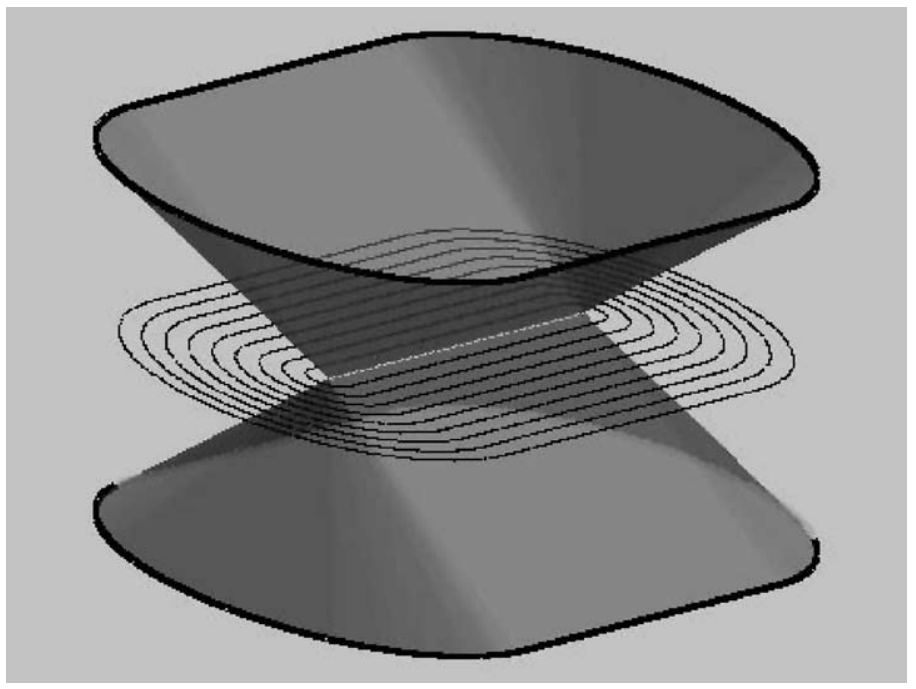

Fig. 5. The solution of the phase diffusion equation over a stadium, is properly defined on a double cover.

it is possible to describe many of these solutions in terms of a well defined, continuous single-valued $\theta$ (at least away from the point defects), these descriptions involve requiring sharp "ridges", where $\vec{k}=\nabla \theta$ flips direction by $180^{\circ}$. The energetic penalty for such a switch would be very high if we consider only vector fields, but, in fact there is no such cost, because there is no difference between $\pm \vec{k}$. The "correct" energy sees no cost associated with these "ridges". The solution whose energy is minimal is one which is not a single-valued function $\theta$ with ridges, but instead lives on some Riemann surface that covers the physical domain with degree 2. An illustration of this kind of "solution" is given in Fig. 5. The figure shows the graph of the double-valued phase $\pm \theta$ for the "stadium solution" whose level curves are plotted on the midplane. The director field, $\pm \vec{k}$, becomes a single-valued vector field on this Riemann surface.

On the other hand, there is remarkably good agreement between the eikonal solutions that we have found in the earlier sections and the computed and experimental solutions on ellipses, except in a neighborhood of a portion of the major axis of the ellipse near the center. Where the eikonal solution predicts that we should have a PGB where rolls bend (fairly sharply), the computed and experimental results show a string of pairs of $\mathrm{V}$ and $\mathrm{X}$ disclinations. These disclinations have a spacing that is large in the center, and becomes closer as we move further out. At a certain point, the string of VX pairs is replaced by a PGB where the rolls behave as in the eikonal solution.

\section{Predicting the critical angle}

Let us now examine the stability of a PGB in a context where the wave vector is a director field and not a vector field. When the roll angle is too sharp, the configuration shown in Fig. 1 is unstable to perturbations in which $(\mathrm{V}, \mathrm{X})$ disclination pairs are created. On the lines joining these, the sign of $\vec{k}$ reverses but such a reversal adds no cost to the free energy. In order to understand this instability, let us return to the pure PGB shown in Fig. 6a. In (4.10) we calculated the asymptotic energy cost per unit length of this grain boundary to be $(8 / 3) \sin ^{3}(\varphi)$. Now, imagine that from the origin on, we change the configuration to that shown in Fig. 6b. We have introduced two new phase grain boundaries, each locally longer than the horizontal PGB by a factor of $1 / \cos (\pi / 4+\varphi / 2)$, but the new angle $\pi / 4-\varphi / 2$ between the $\vec{k}$ vector and the new PGB may be smaller. The free energy per unit length along 
(a)

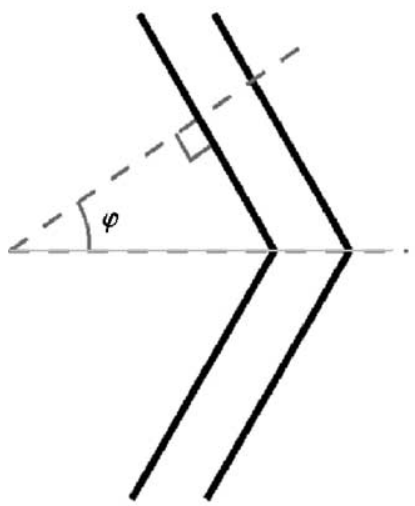

(b)

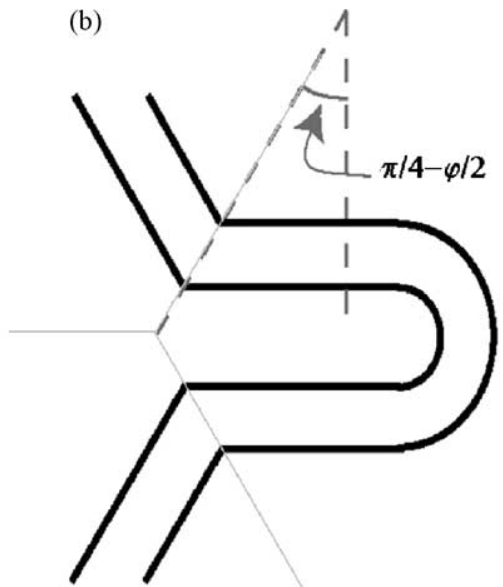

Fig. 6. (a) A single PGB may have higher energy than (b) a configuration of three phase grain boundaries with a pair of disclinations (phase grain boundaries are shown as thin solid gray lines).

the pair of phase grain boundaries shown in Fig. $6 \mathrm{~b}$ is

$$
\begin{aligned}
& \frac{2}{\cos (\pi / 4+\varphi / 2)} \sin ^{3}\left(\frac{\pi}{4}-\frac{\varphi}{2}\right) \frac{8}{3} \\
& =\frac{8}{3}(1-\sin \varphi) .
\end{aligned}
$$

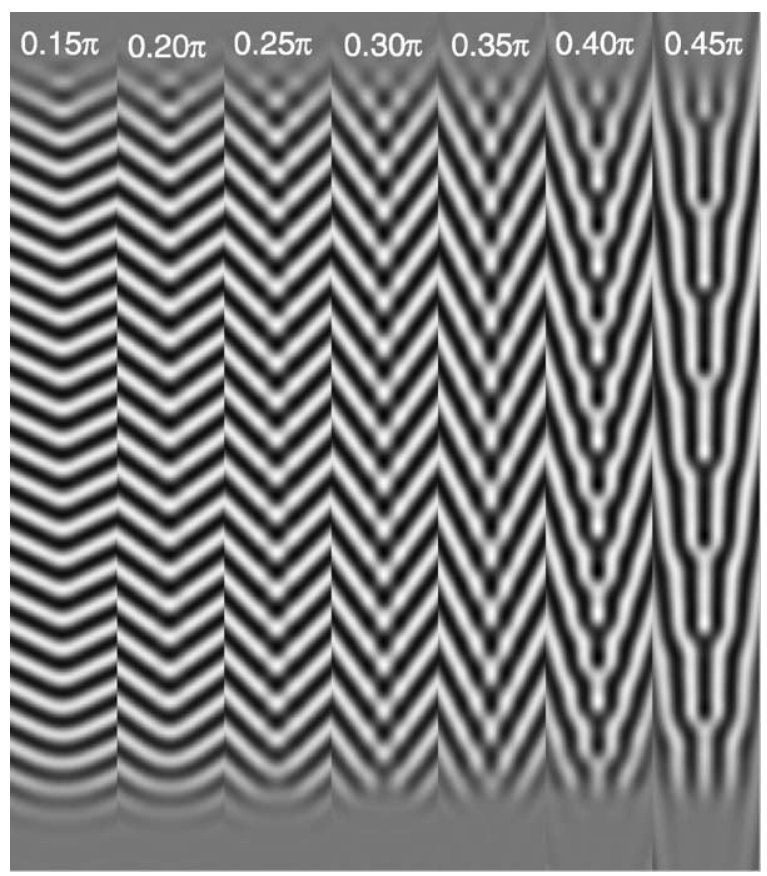

Fig. 7. Solutions of Swift-Hohenberg with boundary conditions forcing the rolls to form at a specified angle, as labeled across the top.
But for large $\varphi, \sin ^{3} \varphi>1-\sin \varphi$; equality occurs at $\varphi_{\mathrm{c}} \simeq 43^{\circ}$. Therefore a PGB configuration whose $\vec{k}$ angle $\varphi$ is too large is expected to destabilize into the configuration shown in Fig. 6b. The vector field solution has destabilized to a director field solution in which a concave-convex disclination pair protuberance has formed. The contribution from the circular end about the convex disclination will be proportional to $\varepsilon \ln (1 / \varepsilon)$ and therefore much smaller than the grain boundary contribution.

In Fig. 7, we show the results of a simple numerical experiment solving the Swift-Hohenberg equation with $R=1$, in which we slowly increase the angle $\varphi$ in a PGB. The roll angles are fixed on the lateral boundary and ramps on the stress parameter are applied at the end parts of the domain. The first appearance of a protuberance indeed appears at an angle $\varphi \approx 45^{\circ}$.

\section{Swift-Hohenberg numerics}

Patterns of very large aspect ratio in an elliptical geometry have been obtained from the numerical integration of the Swift-Hohenberg equation (1.1). We use a pseudo-spectral method in a square periodic domain of width $300 \pi$ with a maximum resolution of $1024^{2}$ grid points. In order to mimic an elliptical boundary condition, the stress parameter $R$ is ramped from its 


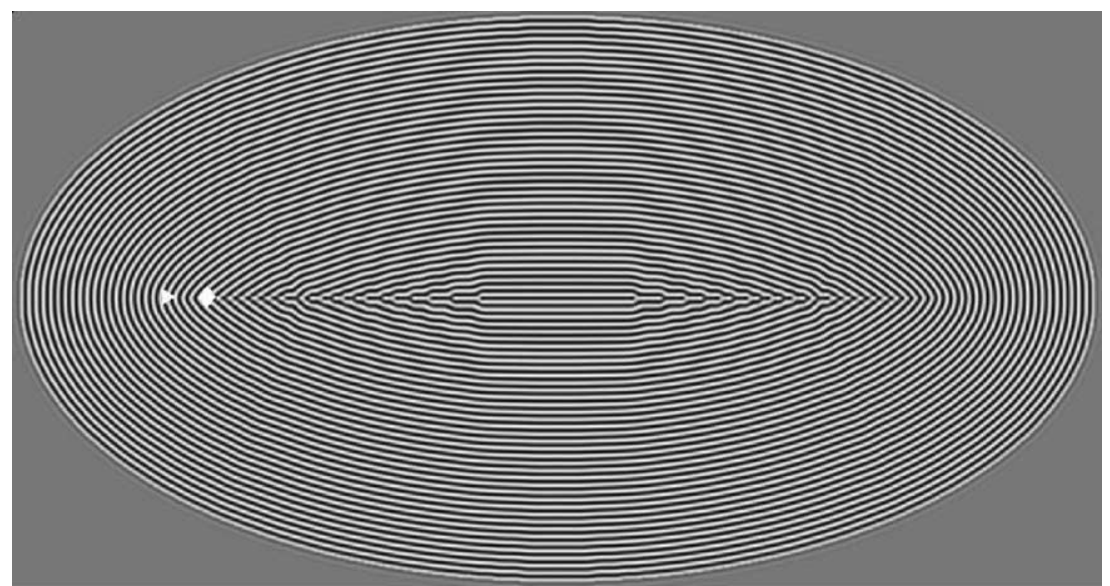

Fig. 8. Rolls from numerical simulation of Swift-Hohenberg for an ellipse with major to minor axis ratio of 1.9. The white triangle marks the center of curvature, the white diamond marks the point where the angle of the PGB in the eikonal solution surpasses the critical angle, as discussed in the text.

value 0.5 inside an ellipse contour whose major axis ends at a distance of five grid points from the sides of the computational domain, to -0.5 outside this contour. The transition takes place within one grid point. Small oscillations are seen outside the ellipse, whose influence is negligible on the dynamics inside the ellipse. The initial conditions are taken to be the cosine of the phase obtained by solving the eikonal equation (5.1) with a zero phase boundary condition on the ellipse boundary. The arbitrary constant that multiplies the solution is chosen to impose $k=1$. This initial condition is displayed in Fig. 1. In Fig. 8 we display the roll pattern for an ellipse where the ratio of major to minor axes equals 1.9 , after a time $t=156000$. At this time, the pattern seems almost stationary, although it is difficult to be absolutely certain that the energy would not continue to decrease. A conspicuous feature of the solution is the straight roll domain in the central part followed by a series of dislocations, all located on the major axis, symmetrically with respect to the origin, which help to match the inner rolls to the outer part of the grain boundary predicted by the theory. This solution differs from that of the weak solution because for the latter, the angle made by the rolls in the central part of the grain boundary is too sharp. In that case, as explained in the previous section, a transition occurs that permits the decrease of the energy by forming pairs of concave and convex disclinations (or dislocations). A diamond shaped mark is inserted on the major axis at the location where theory predicts that the roll angle on the PGB reaches the critical one $\left(43^{\circ}\right)$. It is easy to find this position analytically. For an ellipse of major and minor axes $a$ and $b$, respectively (the eccentricity is then given by $\left.e=\sqrt{\left(a^{2}-b^{2}\right) / a^{2}}\right)$, the angle of the PGB at a position $X$ along the major axis (the origin being at the ellipse center) is given by

$\sin \alpha=\sqrt{\left(1-\frac{a^{2} X^{2}}{\left(a^{2}-b^{2}\right)^{2}}\right) /\left(1-\frac{X^{2}}{a^{2}-b^{2}}\right)}$.

If $\varphi_{\mathrm{c}}$ denotes the critical angle, the position $X_{\mathrm{c}}$ along the major axis of the ellipse where this angle is reached reads

$X_{\mathrm{c}}=\frac{a e^{2}}{\sqrt{1+\left(1-e^{2}\right) \tan ^{2} \varphi_{\mathrm{c}}}}$.

As seen in Fig. 8, the observed position is a good match to the predicted one.

It should be remarked that the terminal disclination which should appear on the major axis at the center of curvature $a e^{2}$, is hardly visible on the SH simulations. 


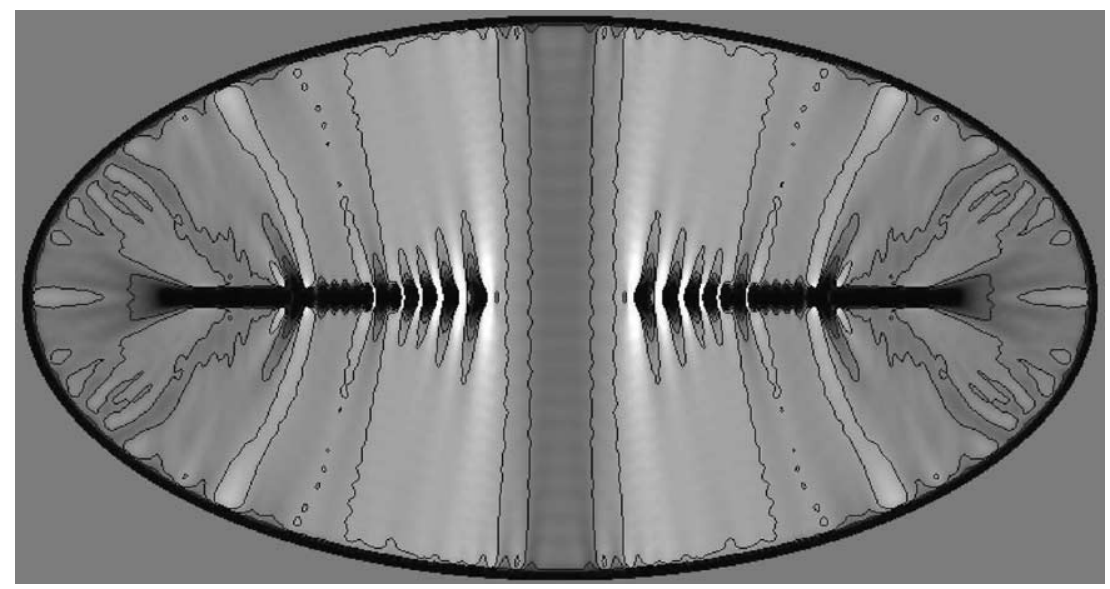

Fig. 9. Local wave number magnitude corresponding to Fig. 9, lighter grays correspond to higher values of $k$. The contours shown are $k=0.995, k=1$ and $k=1.005$.

This is because PGBs here are regularized shocks. Their position is marked with a triangle symbol.

The local wave number, as shown in Fig. 9 reaches a value very close to 1 inside the ellipse, except in the neighborhood of the dislocations. Near the PGBs we see lower values of $k$. Above and below the line of dislocations, $k$ is greater than 1 . (The grayscale in the figure is chosen so that values of $k$ above 1.015 are white, and those below 0.985 are black.) We calculate the local wave number by comparing the magnitude of the gradient of the computed solution of Swift-Hohenberg, to that of computed solutions of Swift-Hohenberg with specified wave numbers over a range near $k=1$. The gradient's magnitude squared is first smoothed by convolution with a bump function with width slightly larger than $2 \pi$ to remove roll scale features, then this value is compared to the values computed in the same way for straight parallel roll solutions of the same equation. This approach is motivated by the ansatz that the solution of $\mathrm{SH}$ can be written as $F(\theta(x, y))$, where $F$ is a periodic function. The result of calculating the squared magnitude of the gradient in this case produces a factor of $k^{2}$ times a term whose average is independent of $k$.

Fig. 10 shows the result of calculating the local energy density for Swift-Hohenberg, and then

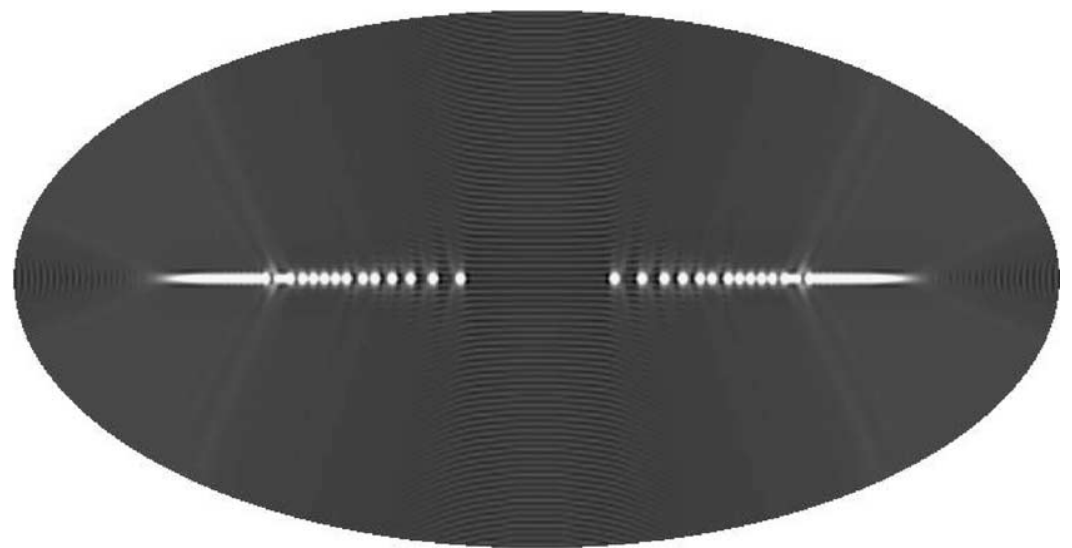

Fig. 10. Smoothed local energy density corresponding to Fig. 8; lighter grays correspond to higher values. 


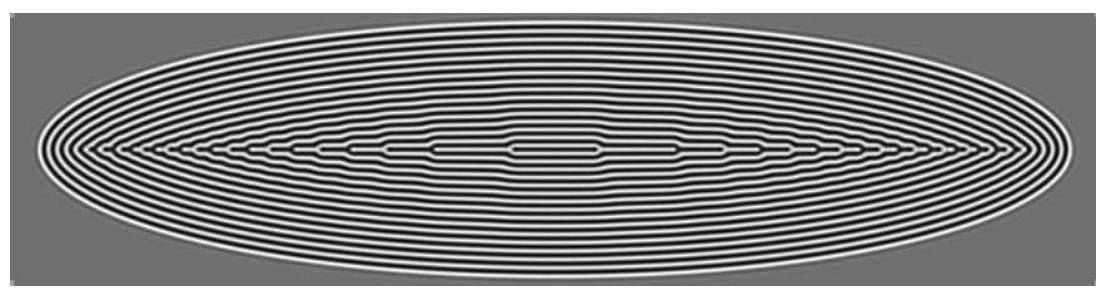

Fig. 11. Rolls from numerical simulation of Swift-Hohenberg with an ellipse whose major axis is four times longer than its minor axis.

smoothing the result by convolving with a bump function to remove features at the scale $\lambda$. The result is not sensitive to the details of the choice of the "bump" function. This energy plot allows a precise localization of the dislocations and shows their gradual packing as the end of the PGB is reached.

Another simulation has been performed in the case where the ratio $a / b=4$. The resulting roll pattern is shown in Fig. 11. Here again, the PGB is replaced by a series of dislocations. It appears that the distance between dislocations depends on the original angle between the rolls at the PGB: tightly spaced dislocations reproduce weak roll bending, while widely spaced ones replace sharply bent rolls.

\section{Comparison to other numerics and experiments}

Similar simulations were performed using the original Oberbeck-Boussinesq equations with top and bottom no-slip boundary conditions [4]. In Fig. 12 we exhibit the roll pattern for such a simulation with a Rayleigh number equal to 2000, a Prandtl number

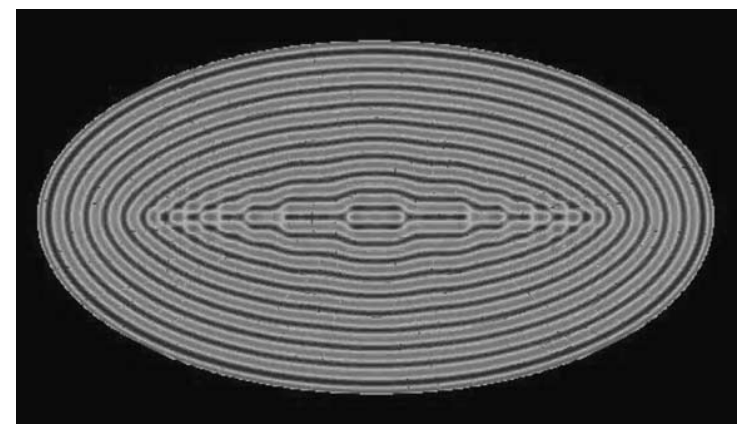

Fig. 12. Roll pattern from numerical simulation of OberbeckBoussinesq equations (courtesy of Paul) [4].
$P=100$ and a ratio of major to minor axes equal to 1.9, as in the first SH simulation [4]. Sidewalls are heated in order to maintain rolls parallel to the elliptical boundary. A remarkable agreement is found with the SH simulations, indicating that the latter model reproduces the principal features of the solution.

Experiments have also been performed [5] in ethanol at a Prandtl number of 14.7. Although the inverse aspect ratio is not as small as in the previous simulations, the same features are again observed, showing the robustness of the arguments based on the phase diffusion equation. In particular one clearly sees the protuberances at the end of the inner rolls.

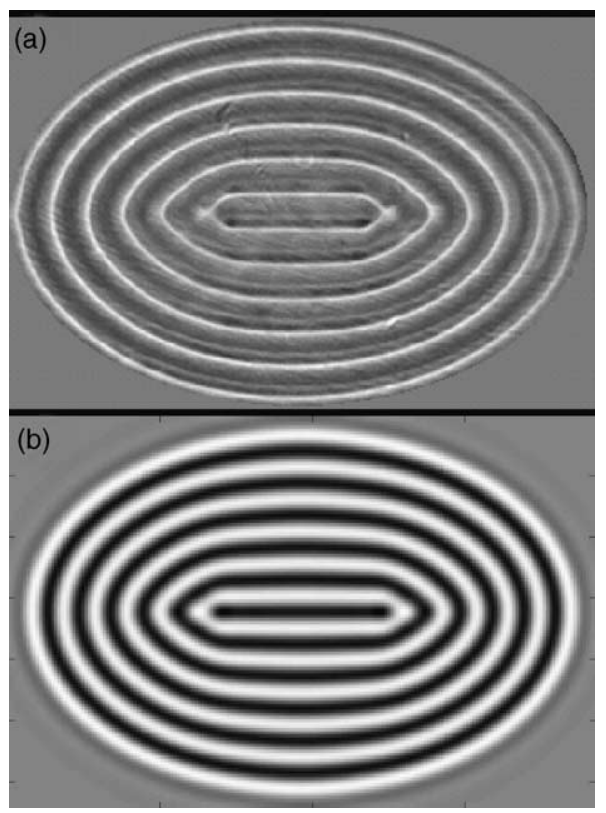

Fig. 13. (a) Image of convection rolls from an experiment with ethanol (courtesy of Meevasana and Ahlers [5]). (b) Numerical simulation of Swift-Hohenberg with similar geometry. 


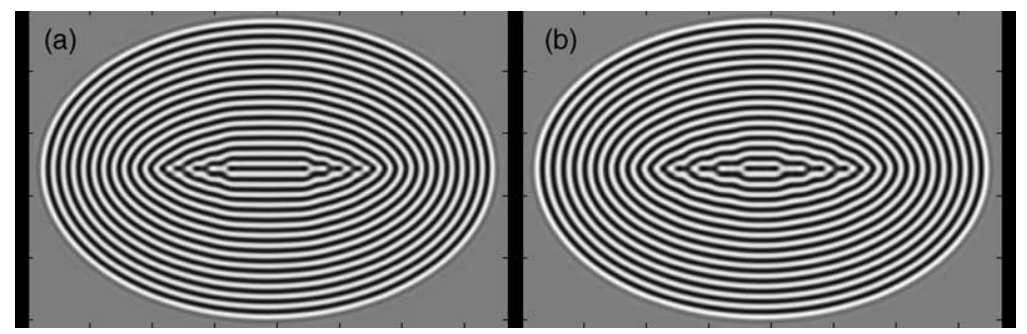

Fig. 14. (a) Result of integrating Swift-Hohenberg using $k_{\mathrm{B}}=1$. This was used as the initial condition for (b) which was Swift-Hohenberg for $k_{\mathrm{B}}=0.95$.

Dislocations do not form because of the large aspect ratio. An SH simulation has been performed with the same number of rolls and the same eccentricity (ratio $a / b=1.5)$. A comparison is shown in Fig. 13. In addition, it is found in the experiments [5] that the size of the inner straight roll decreases as the stress parameter is increased. This particular result is not found with the SH equation. This behavior is likely to be related to the decrease of the selected wave number in Oberbeck-Boussinesq as the Rayleigh number increases. The only direction where this adjustment can take place without roll annihilation is along the major axis. It is achieved by reducing the length of the inner roll, at the expense of a deviation from the selected wave number at other places. This can happen as long as the local wave number does not fall outside the Busse balloon. When it does, a pair of dislocations form [5], breaking the up-down symmetry. This hypothesis has been tested with the $\mathrm{SH}$ equation in the following manner: using as initial conditions the results of a simulation where the selected wave number is exactly 1 , we integrated the $\mathrm{SH}$ with a linear operator modified in such a way as to select $k=$ 0.95. As predicted the inner rolls shrank as depicted in Fig. 14.

\section{Conclusion}

In this paper we have presented strong numerical and experimental evidence that the correct variational model for pattern formation far from threshold is a modification of the regularized Cross-Newell phase diffusion equation that permits variations over a class of vector fields which are only constrained to be $l o-$ cally gradient. Moreover, we have been able to analytically study a local comparison between the energy cost for systems which do or do not count the cost of an orientational reversal. Based on this we make a prediction of the transition angle at which a PGB will destabilize into a chain of concave-convex disclination pairs or dislocations. This prediction is seen to be well supported in numerical simulations and experiments. In other words the energy should be modified to discount the bending energy cost associated with sign reversal of the local wave vector. Such a model is currently under investigation and will be reported in future work.

It is worth noting that there is an explicit formula for a string of dislocations whose character is quite similar to the dislocations seen in Figs. 7, 8 and 11. For example, a string of dislocations located along the $x$-axis, at positions $\pm x_{n}$ is

$$
\begin{aligned}
\theta= & y-\operatorname{sign}(y) \epsilon \log \left(1+\sum_{n=1}^{N}\left(\frac{\mathrm{e}^{n \pi}-\mathrm{e}^{(n+1) \pi}}{2}\right)\right. \\
& \times \operatorname{erf}\left(\frac{x-x_{n}}{\sqrt{2 \epsilon|y|}}\right)-\sum_{n=1}^{N}\left(\frac{\mathrm{e}^{n \pi}-\mathrm{e}^{(n+1) \pi}}{2}\right) \\
& \left.\times \operatorname{erf}\left(\frac{x+x_{n}}{\sqrt{2 \epsilon|y|}}\right)\right) .
\end{aligned}
$$

This is an exact solution of the phase equation with a "weak bending" assumption [11]. In fact, if a multi-dislocation with spacing seen in Fig. 8 is patched to the outer portion of the eikonal solution, this initial condition settles to a solution of 
the same form with the position of the dislocations essentially unchanged. (The only real changes are along the interface where the solutions are patched together.)

Another natural extension of the RCN problem is to study minimizers of the free energy

$\mathcal{F}=\int_{\Omega}\left(|\nabla \vec{k}|^{2}+\frac{1}{\varepsilon^{2}}\left(k^{2}-1\right)^{2}+\frac{1}{\mu^{2}}(\nabla \times \vec{k})^{2}\right) \mathrm{d} \vec{X}$

in the limits $\varepsilon, \mu \rightarrow 0$. We note that the case where $\varepsilon$ is set to infinity and only limits in $\mu$ are considered corresponds to a natural model [13] for micro-magnetic systems whose asymptotic minimizers are identical to those described in [1]. Such a study may also have implications for models of defects in superconductors [14] and nematic liquid crystals [15].

\section{Acknowledgements}

The authors wish to thank Joceline Lega for many helpful discussions and in particular for generating Fig. 3. The authors also wish to thank Guenter Ahlers and Mark Paul for helpful discussion and for generosity in sharing their results. NME would like to acknowledge support from NSF grant DMS-0073087 and $\mathrm{ACN}$ would like to acknowledge support from NSF grant DMS-0072803.

\section{References}

[1] N.M. Ercolani, R. Indik, A.C. Newell, T. Passot, The geometry of the phase diffusion equation, J. Nonlinear Sci. 10 (2000) 223-274.

[2] M.C. Cross, P.C. Hohenberg, Pattern formation outside of equilibrium, Rev. Mod. Phys. 65 (1993) 851-1112.

[3] Y. Pomeau, Caustics of nonlinear waves and related questions, Europhys. Lett. 11 (1990) 713-718.

[4] M. Paul, Private communication.

[5] W. Meevasana, G. Ahlers, Private communication.

[6] F.H. Busse, On the stability of two-dimensional convection in a layer heated from below, J. Math. Phys. 46 (1967) 149-150.

[7] M.C. Cross, A.C. Newell, Convection patterns in large aspect ratio systems, Physica D 10 (1984) 299-328.

[8] L. Ambrosio, C. DeLellis, C. Mantegazza, Line energies for gradient vector fields in the plane, Calc. Var. 9 (1999) 327355.

[9] N.M. Ercolani, M. Taylor, The Dirichlet-to-Neumann map, viscosity solutions to eikonal equations, and the self-dual equations of pattern formation, Preprint, 2002.

[10] A. DeSimone, R.V. Kohn, S. Muller, F. Otto, A compactness result in the gradient theory of phase transitions, Proc. R. Soc. Edinburgh Sect. A 131 (2001) 833-844.

[11] A.C. Newell, T. Passot, C. Bowman, N.M. Ercolani, R. Indik, Defects are weak and self-dual solutions of the Cross-Newell phase diffusion equation for natural patterns, Physica D 97 (1996) 185-205.

[12] T. Passot, A.C. Newell, Towards a universal theory of patterns, Physica D 74 (1994) 301-352.

[13] W. Jin, R. Kohn, Singular perturbation and the energy of folds, J. Nonlinear Sci. 10 (2000) 355-390.

[14] A. Jaffe, C. Taubes, Vortices and Monopoles, Birkhauser, Boston, 1980.

[15] F.H. Lin, Nonlinear theory of defects in nematic liquid crystals; phase transition and flow phenomena, Commun. Pure Appl. Math. 42 (1989) 789-914. 